\title{
Improved radiosensitizing effect of the combination of etanidazole and paclitaxel for hepatocellular carcinoma in vivo
}

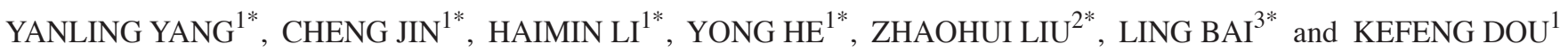 \\ ${ }^{1}$ Department of Hepatobiliary Surgery, Xijing Hospital, Fourth Military Medical University, Xi'an 710032; \\ ${ }^{2}$ Department of Rehabilitation Medicine, Tangdu Hospital, Fourth Military Medical University, Xi'an 710038; \\ ${ }^{3}$ Department of Clinical Laboratories, Xi'an Gaoxin Hospital, Xi'an 710075, P.R. China
}

Received September 13, 2011; Accepted October 31, 2011

DOI: $10.3892 /$ etm.2011.389

\begin{abstract}
Hepatocellular carcinoma (HCC) is one of the most critical global health issues. Potential curative therapies, including surgical resection, are offered to only a limited number of patients. Therefore, new and effective treatment strategies are required. Recently, radiotherapy with hypoxic radiosensitizers has shown promise in cancer therapy. Our previous study demonstrated that radiosensitization produced by etanidazole and paclitaxel was additive in vitro. This study was carried out to determine the synergistic effect of the two drugs in murine HCC H22 cell xenograft-bearing BALB/c mice in vivo. The morphology of the transplanted tumors was observed. The drug content in the blood and tumors of mice was measured by high-performance liquid chromatography. The radiosensitizing effect on $\mathrm{H} 22$ cell xenograft-bearing mice was evaluated in terms of tumor growth inhibition and survival. Expression of hypoxia inducible factor-1 $\alpha$ (HIF-1 $\alpha)$ was studied using immunohistochemistry. The morphological consequences on the $\mathrm{H} 22$ xenografts were consistent with the pathological characteristics of HCC. There was no significant difference in drug content in the blood and tumors between single drug and combination administration. The combination of the two drugs improved the radiosensitizing effect in vivo compared to single drug administration in an animal model. The changes in HIF-1 $\alpha$ expression indirectly verified the above-mentioned results. This study may provide a new combination of radiosensitizers for HCC radiotherapy.
\end{abstract}

Correspondence to: Professor Kefeng Dou or Dr Cheng Jin, Department of Hepatobiliary Surgery, Xijing Hospital, Fourth Military Medical University, Xi'an 710032, P.R. China

E-mail: kefengdou@tom.com or jc4587@tom.com

${ }^{*}$ Contributed equally

Key words: hepatocellular carcinoma, paclitaxel, etanidazole, combination radiosensitization

\section{Introduction}

Hepatocellular carcinoma (HCC) is one of the critical global health issues. As indicated in cancer statistics, HCC is the sixth most common type of tumor worldwide, but due to its poor prognosis it ranks as the third most common cause of mortality from cancer $(1,2)$. Despite therapeutic advances, the overall survival of patients with HCC has not significantly improved in the last two decades. Surgical resection, radiofrequency ablation (RFA) and percutaneous ethanol injection (PEI) are curative treatments; however, they are accompanied by high recurrence rates (3-5). Transarterial chemoembolization (TACE), the most popular non-surgical alternative, is often used as a palliative treatment (6). General chemotherapy and radiotherapy offer somewhat unsatisfactory responsiveness. Thus, new therapeutic strategies are necessary to combat HCC.

Based on observations from the beginning of the last century, it has become well established that solid tumors may contain oxygen-deficient hypoxic areas, and that cells in such areas may cause tumors to become radioresistant $(7,8)$. A substantial amount of data have indicated the presence of hypoxia in numerous types of human tumors, including HCC. Treating hypoxic tumor cells could involve the specific modification of tumor radiosensitivity by the use of chemical radiosensitizers. Targeting various physiological characteristics of the tumor, radiosensitizers are intended to enhance tumor cell killing by radiation, while having much less of an effect on normal tissues $(9,10)$. Etanidazole, a nitroimidazole hypoxic radiosensitizer, has the advantage of lower toxicity at gram-level doses administered to patients in order to attain tumor concentrations reasonable for radiosensitization (11). Paclitaxel is one of the best anti-neoplastic drugs discovered in previous decades. A number of studies have confirmed the radiosensitizing effect of paclitaxel (12). Owing to the side-effects of radiosensitizers, clinical protocols frequently combine these drugs, which exhibit their cytotoxic action at various mechanisms. This approach minimizes the overall toxicity while enhancing maximal radiosensitization (13). This strategy has been used to study the radiosensitizing effect of the combination of etanidazole and paclitaxel in our previous study. The results indicated that the radiosensitization produced by etanidazole and paclitaxel was additive in vitro $(14,15)$. 
This study was carried out to evaluate the radiosensitizing effect of the combination of etanidazole and paclitaxel for $\mathrm{HCC}$ in vivo, using a murine $\mathrm{H} 22$-bearing BALB/c model.

\section{Materials and methods}

Drugs. Etanidazole was purchased from Sigma-Aldrich (St. Louis, MO, USA). Paclitaxel (H20063662, $30 \mathrm{mg} / 5 \mathrm{ml}$ ) was from Beijing Sihuan Pharmaceutical Co. Ltd (Beijing, China). Etanidazole was dissolved in phosphate-buffered saline (PBS; pH 7.4) at a concentration of $10 \mathrm{mg} / \mathrm{ml}$ for the actual test. A paclitaxel stock solution of $10 \mu \mathrm{g} / \mathrm{ml}$ prepared in PBS medium was maintained at $-20^{\circ} \mathrm{C}$ and thawed for use.

Cell culture. The murine HCC H22 cells were purchased from the Experimental Animal Center of the Fourth Military Medical University (Xi'an, China). The cells were frozen and stored in liquid nitrogen for further use. For experiments, the cells were thawed and subcultured in ascites of $\mathrm{BALB} / \mathrm{c}$ mice.

Animals. Male BALB/c mice $(20 \pm 2 \mathrm{~g})$ were supplied by the Experimental Animal Center of the Fourth Military Medical University. The animals were acclimatized at a temperature of $25 \pm 2^{\circ} \mathrm{C}$ and a relative humidity of $70 \pm 5 \%$ under natural light/dark conditions for 1 week prior to dosing. All animal experiments were performed under the guidelines of an approved protocol from the Committee on the Use of Live Animals in Teaching and Research of the Fourth Military Medical University.

Radiation. Radiation was delivered at room temperature utilizing a ${ }^{60} \mathrm{Co}$ source (Department of Radiation Medicine, Fourth Military Medical University). The dose rate depended on the distance from the source. Radiation was delivered at $266.82 \mathrm{cGy} / \mathrm{min}$ for a duration of $1 \mathrm{~min} 52 \mathrm{sec}$ to produce a dose of $5 \mathrm{~Gy}$. Following radiation, animals were maintained under standard breeding conditions for 6 months.

Tumor morphology. $\mathrm{H} 22$-bearing BALB/c mice were studied. In order to establish the murine HCC xenografts, a cell suspension $(0.2 \mathrm{ml})$ containing $2 \times 10^{6} \mathrm{H} 22$ cells was inoculated subcutaneously into the back of the mice for 12 days. The mice were sacrificed by cervical dislocation and tumors were then removed. Sections of tumor tissue were fixed in neutral buffered formalin and the paraffin sections were prepared for hematoxylin and eosin (H\&E) histological staining. The photomicrography was performed using a Nikon TE2000-S microscope (Japan).

Drug content in blood and tumors. Drug content in the blood and tumors of H22-bearing mice $(n=5)$ was evaluated by high-performance liquid chromatography (HPLC). Etanidazole at $200 \mathrm{mg} / \mathrm{kg}$ and/or paclitaxel at $1 \mathrm{mg} / \mathrm{kg}$ were injected through the tail vein 12 days following inoculation. The mice were treated with PBS as the control. Two hours following administration, the mice were sacrificed by cervical dislocation. Blood was then collected via decapitation and tumors were removed. Blood was placed at room temperature for $30 \mathrm{~min}$ and then centrifuged at 1,000 rpm for $5 \mathrm{~min}$. Blood serum was collected.

A total of $200 \mathrm{mg}$ of tumors were homogenized in $3 \mathrm{ml}$ of a mixture of acetonitrile: water [50:50, $(\mathrm{v} / \mathrm{v})]$ or acetonitrile:water
[5:95, (v/v)] and centrifuged at 3,000 rpm for $5 \mathrm{~min}$. The supernatant was taken for analysis of drug content by HPLC.

A total of $200 \mu \mathrm{l}$ of blood serum were mixed with $1 \mathrm{ml}$ of a mixture of acetonitrile:water [50:50, (v/v)] or acetonitrile:water $[5: 95,(v / v)]$. The solution containing the drugs was determined using HPLC.

The HPLC assay (Agilent 1100 series) for etanidazole and paclitaxel was performed on a reverse phase Zorbax ${ }^{\circledR} \mathrm{C} 18$ column. The mobile phases for etanidazole and paclitaxel were the mixtures of acetonitrile: water 5:95 and 50:50 (v/v), respectively. The mobile phase was delivered at a flow rate of $1.0 \mathrm{ml} / \mathrm{min}$. Etanidazole and paclitaxel were detected at 324 and $227 \mathrm{~nm}$, respectively, with a variable wavelength detector. The calibration curves for the quantification for etanidazole and paclitaxel were linear over the range of a standard concentration between 50 and $100,000 \mathrm{ng} / \mathrm{ml}$ with a correlation coefficient of $\mathrm{R}^{2}=0.99$ and 1.00 .

Radiosensitizing effect in vivo. Animals were randomized 12 days following inoculation and divided into 8 mice/group (control, radiation only, etanidazole, paclitaxel, and combination of etanidazole and paclitaxel). The administration of drugs and radiation were conducted as described above. Radiation was performed 2 hours following administration. The mice received a single dose of whole-body $\gamma$-ray radiation using a ${ }^{60} \mathrm{Co}$ source. Mice were monitored daily and all surviving mice were sacrificed on day 180. Tumor volumes [(major axis) x (minor axis $)^{2} \times 1 / 2$ ] were measured with a caliper at defined time periods for 40 days post-radiation. The tumor inhibition rate (\%) was calculated using the following formula: (tumor volume of control - tumor volume of experiment)/tumor volume of control x $100 \%$.

Immunohistochemistry. The expression of hypoxia inducible factor-1 $\alpha$ (HIF-1 $\alpha)$ was determined by immunohistochemistry staining. Two days following radiation, H22-bearing mice were sacrificed and the tumors were removed. Paraffin-embedded tissue sections at $4 \mu \mathrm{m}$ were prepared. Tissue sections were deparaffinized in xylene and rehydrated in graded alcohols and distilled water. Slides were processed for antigen retrieval by a standard microwave heating technique in citrate buffer ( $\mathrm{pH}$ 6.0). Endogenous peroxidases were inactivated by immersing the sections in $0.3 \%$ hydrogen peroxide for $10 \mathrm{~min}$ twice. The primary polyclonal rabbit antibody reacts specifically with mouse HIF-1 $\alpha$ (dilution 1:100; Wuhan Boster Biological Technology Co. Ltd, China). The sections were incubated with the antibody overnight at $4^{\circ} \mathrm{C}$ in a humidified chamber. Immunodetection was performed using a standard avidin-biotin peroxidase technique. The reaction was then developed using the Liquid DAB Substrate-Chromogen System. Sections were counterstained with Mayer's hematoxylin. Positive staining (brown) for HIF-1 $\alpha$ proteins was observed predominantly in the cytoplasm of cells. More than 1,000 cells in 5-6 various high-power fields (x400) were analyzed for each section.

Statistical analysis. Comparisons of the drug content and tumor volume were performed using one-way ANOVA. Survival studies were analyzed using Kaplan-Meier plots. The Chi-square test was used to compare the positive rate of HIF-1 $\alpha$. All analyses were performed using the statistical software SPSS10.0. 
A

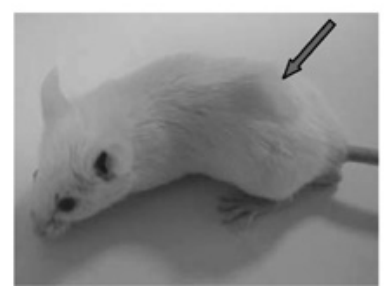

B

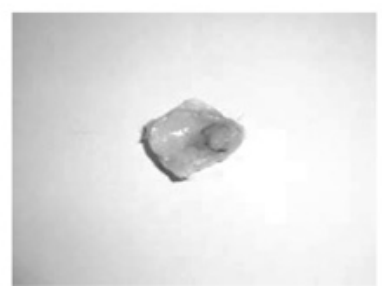

C

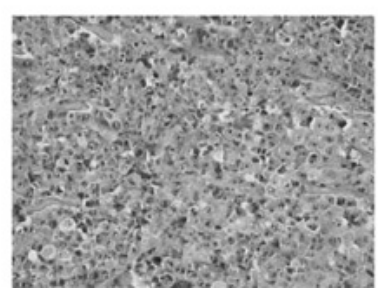

Figure 1. (A) A mouse bearing an H22 xenograft. The tumor nodule is indicated by the red arrow. (B) The tumor was retrieved. (C) Tumor tissue H\&E stain, $\mathrm{x} 400$.

A

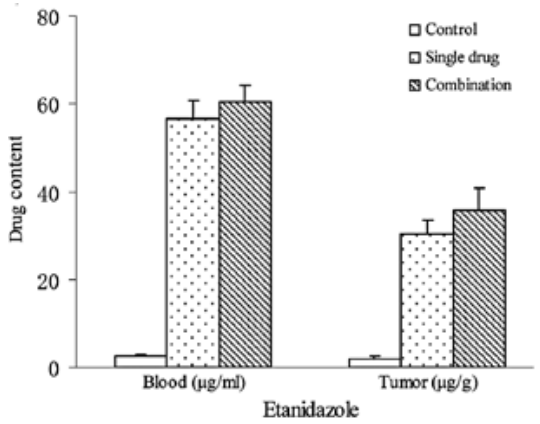

B

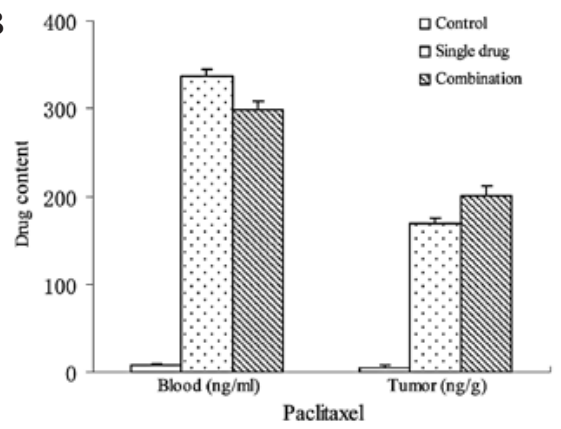

Figure 2. Drug content in the blood and tumors $2 \mathrm{~h}$ following intravenous administration. (A) Etanidazole. (B) Paclitaxel.

\section{Results}

Tumor morphology. The morphological changes of $\mathrm{H} 22$ xenografts are shown in Fig. 1. When treatments were commenced on day 12 post-incubation, tumor size was approximately $0.2 \mathrm{~cm}^{3}$. Macroscopic forms of the tumors were grayish-white and soft. In microscopic appearance, the neoplastic cells still resembled normal hepatocytes, but the nuclei were large and more hyperchromatic. Moreover, the nuclei were more prominent than the cytoplasm. This was consistent with the pathological characteristics of HCC.

Drug content in blood and tumors. Drug content in the blood and tumors of H22-bearing mice $2 \mathrm{~h}$ after intravenous administration is shown in Fig. 2. For etanidazole and paclitaxel, there was no significant difference in drug content in the blood or tumors between treatment with either drug alone or combination treatment. Etanidazole content in the blood was $56.7 \pm 4.1$ and $60.5 \pm 3.8 \mu \mathrm{g} / \mathrm{ml}$ for treatment with etanidazole alone and combination treatment, respectively. Etanidazole content in the tumors was $30.2 \pm 3.3 \mu \mathrm{g} / \mathrm{m}$ and $35.6 \pm 5.1 \mu \mathrm{g} / \mathrm{g}$ for treatment with etanidazole alone and combination treatment, respectively. Paclitaxel content in the blood was $336.9 \pm 7.8$ and $229.5 \pm 8.4 \mathrm{ng} / \mathrm{ml}$ for treatment with paclitaxel alone and combination treatment, respectively. Paclitaxel content in the tumors was $168.7 \pm 6.9 \mathrm{ng} / \mathrm{m}$ and $201.3 \pm 10.4 \mathrm{ng} / \mathrm{g}$ for treatment with paclitaxel alone and combination treatment, respectively.

Radiosensitizing effect in vivo. To assess the radiosensitizing effect in vivo, etanidazole and/or paclitaxel were injected through the tail vein of H22-bearing mice at clinically relevant doses. Following administration, the mice were exposed to radiation at 5 Gy. Tumor regression was observed in treated mice (Fig. 3).
It clearly shows that the combination of the two drugs had the greatest regressional effect on the tumor burden in all groups. Forty days following radiation, the tumor inhibition rates (\%) were $19.2,33.9,54.8$ and $61.6 \%$ for radiation only, etanidazole, paclitaxel, and combination therapy, respectively. In addition, there was a significant difference in the survival rate for these treatments. Observable even up to 180 days, the animals displayed $0,0,0,12.5$ and $25.0 \%$ survival rates for control, radiation only, etanidazole, paclitaxel, and combination therapy, respectively. The median survival times were $31.0 \pm 8.5,55.0 \pm 3.5,56.0 \pm 7.1$, 89.0 \pm 7.1 and 108.0 \pm 31.1 days for the control, radiation only, etanidazole, paclitaxel, and combination therapy, respectively (Fig. 4).

Immunohistochemistry. The expression of HIF-1 $\alpha$ in H22 xenografts was demonstrated immunohistochemically by positive cytoplasmic staining (Fig. 5). Its expression was observed in all samples and the percentages of positively stained cells were $63.7,49.2,43.6,24.3$ and $13.6 \%$ for the control, radiation only, etanidazole, paclitaxel, and combination therapy, respectively. HIF-1 $\alpha$ expression in the treated groups was significantly lower than in the control group. Moreover, HIF-1 $\alpha$ expression in the paclitaxel and combination groups was lower than in the radiation only and etanidazole-treated groups.

\section{Discussion}

Although the development of imaging modalities has made the early diagnosis of HCC possible, surgically resectable cases are relatively rare owing to a hepatic function reserve and/or an advanced stage at presentation. Several modalities, including RFA, PEI, TACE and microwave coagulation therapy (MCT) reportedly aid the treatment of patients with unresectable disease. However, unfortunately, it is effective only on limited occasions. 


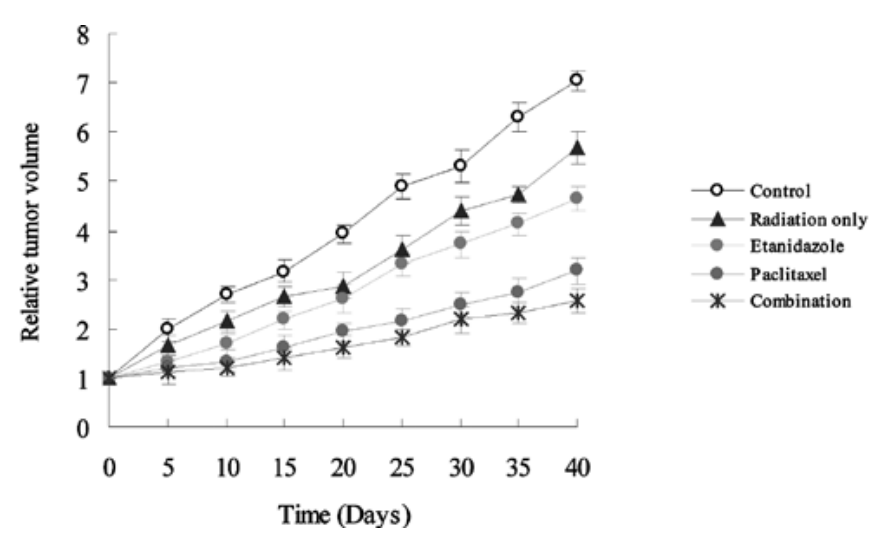

Figure 3. Anti-tumor effects (in terms of tumor growth) of PBS (control), radiation only, etanidazole, paclitaxel, and combination of paclitaxel and etanidazole. PBS, phosphate-buffered saline.

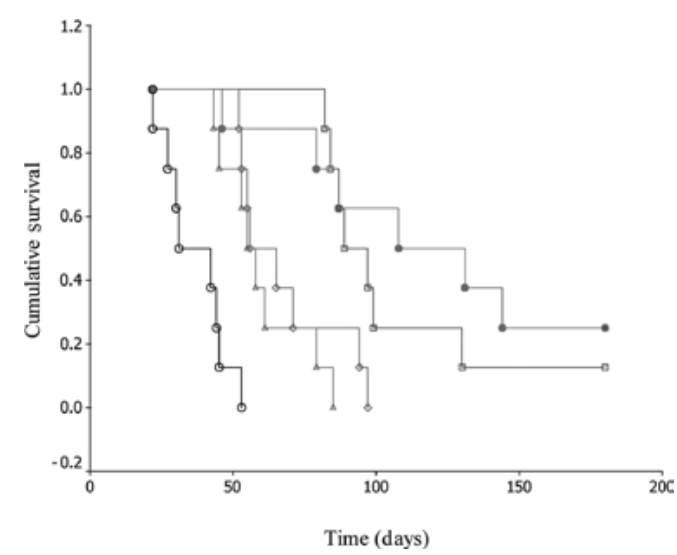

Figure 4. Survival studies of mice bearing H22 xenografts. Survival curves were derived from groups of eight animals each. Open circle, control; open triangle, radiation only; open diamond, etanidazole; open square, paclitaxel; closed circle, combination of paclitaxel and etanidazole.

Therefore, intensive research efforts have been directed toward the identification of novel treatment strategies for HCC.

Radiotherapy technology has evolved markedly over the past decade, and radiation is capable of being precisely delivered, thereby permitting higher doses to the tumor and reduced doses to surrounding normal tissues. There has been increasing interest in the merits of radiotherapy in HCC over the past few years. Radiotherapy has been used as the definitive therapy with curative intent in early stage tumors. It has also been used in combination with TACE for intermediate stage tumors. In locally advanced tumors, radiotherapy has been combined with systemic agents, including chemotherapeutic drugs and hypoxic radiosensitizers (16-18). Certain studies have shown promising survival outcomes. Our previous study indicated that the combination of etanidazole and paclitaxel had a synergetic radiosensitizing effect on MCF-7 and HeLa cells in vitro $(14,15)$. Based on these findings, we therefore investigated the efficacy of the combination of the two drugs in vivo for overcoming $\mathrm{HCC}$.

In the present study, the morphology of $\mathrm{H} 22$ xenografts in macroscopic and microscopic forms displayed HCC characteristics. This demonstrates that the transplantation of tumors
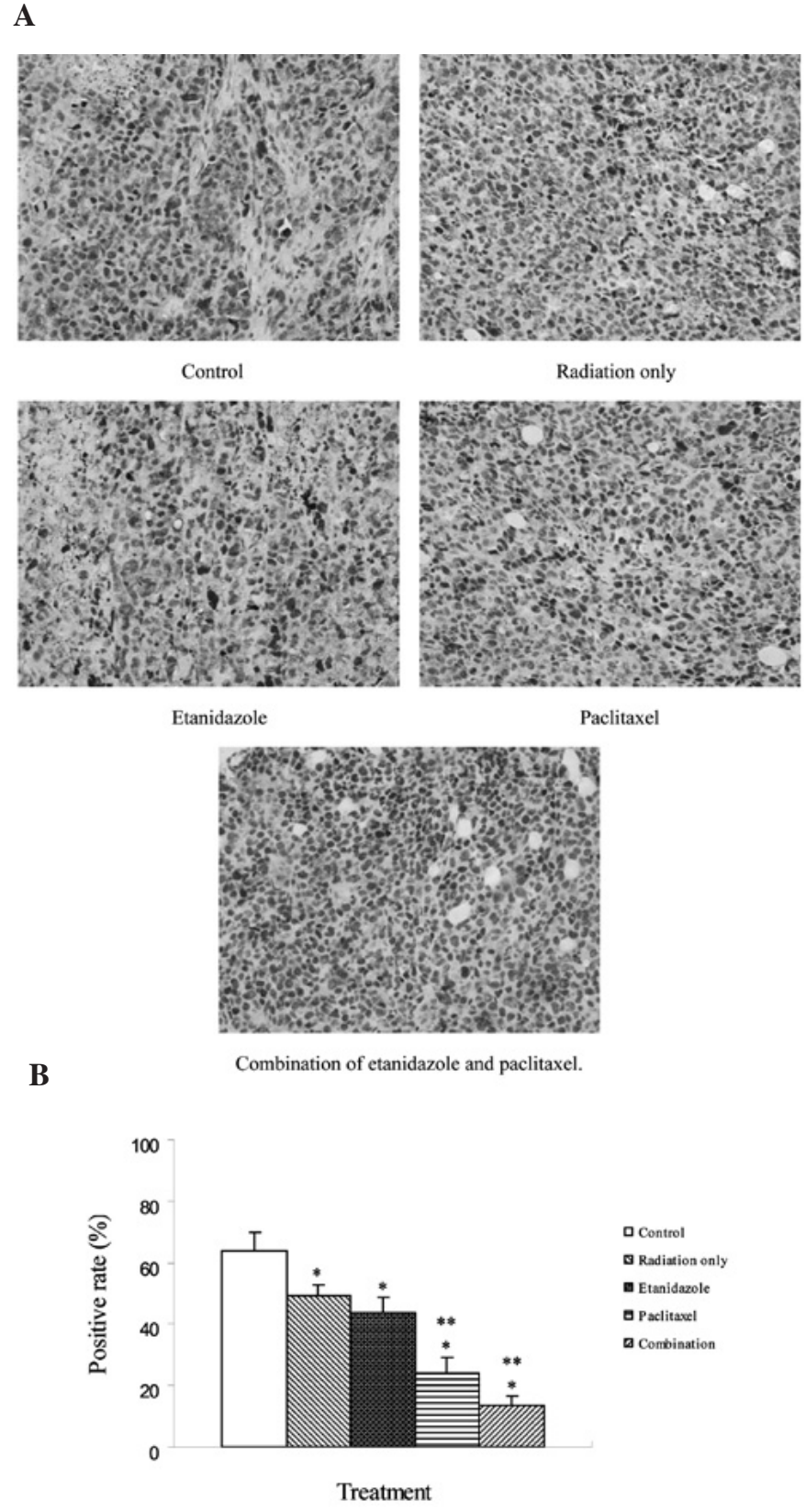

Figure 5. Expression of HIF-1 $\alpha$ proteins in $\mathrm{H} 22$ cell-derived tumor nodules following treatment. (A) Immunohistochemical staining, $x 400$. (B) Positive rate of HIF- $1 \alpha$ in various groups. ( $\mathrm{P}<0.05$ vs. control, ${ }^{* * *} \mathrm{P}<0.05$ vs. radiation only and etanidazole).

was successfully carried out, and lays the foundation for further study.

The HPLC assay was used to determine drug content in blood and tumors of mice bearing $\mathrm{H} 22$ xenografts. Our results indicated that there was no significant difference in content of the two drugs between treatment with either drug alone or in combination. This eliminated the effect of the difference in drug content on therapeutic efficacy.

To understand the synergistic radiosensitizing effect of the two drugs in vivo, a survival assay was performed. Overall, the combination of the two drugs exhibited an improved therapeutic profile in terms of tumor growth inhibition and survival, compared to single drug administration in animal models. 
Clearly, the treatment with etanidazole and/or paclitaxel resulted in more significant tumor regression than radiation therapy alone. The effect of paclitaxel was more significant than that of etanidazole at these administered doses. These data demonstrate that the combination of the two drugs has a synergistic effect. These results are consistent with our previous study in vitro. The lifespan of H22-bearing mice treated with radiosensitizers was significantly longer than the control and mice treated with radiation only. Animals treated with a combination of the two drugs exhibited the longest lifespan in all the groups and had a markedly improved survival rate. Therefore, the co-administration of the two drugs may result in a beneficial gain in HCC radiotherapy. However, complete tumor regression was not observed. This suggests that the administration dose may be modified to produce an improved radiosensitizing effect in future studies.

HIF-1 is as a key transcriptional mediator of the hypoxic response in tumor cells, regulating the expression of a myriad of genes involved in oxygen transport, glucose uptake, and glycolysis and angiogenesis. The overexpression of HIF-1 is observed in the zones of avascularity and hypoxia of most tumors. As a significant marker, HIF-1 expression increases with hypoxic level in tumor location $(19,20)$. Therefore, it may indirectly represent the number of hypoxic cells. This study indicated that treatment with etanidazole and/or paclitaxel resulted in a decrease in the number of hypoxic cells and that a combined treatment was most significant. It further verified the results of the survival assay.

In brief, this study demonstrates the efficacy of radiosensitization by the combination of etanidazole and paclitaxel in vivo for HCC. The data suggest the possibility of its clinical application. However, to date, there is no report that the radiosensitizing effect of the combination is additive clinically. Therefore, well-designed prospective studies are strongly recommended to provide evidence of the true efficacy.

In conclusion, etanidazole and paclitaxel demonstrated the ability to radiosensitize the HCC cells in vivo. The radiosensitizing effect produced by the two drugs was additive when they were administered together at clinically relevant concentrations. This study may provide a new combination of drugs for HCC radiotherapy, although further clinical studies are required

\section{Acknowledgements}

This study was supported by grants from the National Nature Science Foundation of China (No. 81000987) and the China Postdoctoral Science Foundation Funded Project (No. 201003744).

\section{References}

1. World Health Organization. Mortality database. WHO statistical information system. http://www.who.int/whosis/. Accessed July 2008.

2. Parkin DM, Bray F, Ferlay J and Pisani P: Global cancer statistics, 2002. CA Cancer J Clin 55: 74-108, 2005.

3. Padma S, Martinie JB and Iannitti DA: Liver tumor ablation: percutaneous and open approaches. J Surg Oncol 100: 619-634, 2009.

4. Ichida T, van Thiel DH and Hassanein T: The medical management of hepatocellular carcinoma (HCC) in Japan: a review with implications for HCC seen in the west. Hepatogastroenterology 43: 1575-1583, 1996.

5. Bruix J, Sherman M; Practice Guidelines Committee, American Association for the Study of Liver Diseases: Management of hepatocellular carcinoma. Hepatology 42: 1208-1236, 2005.

6. Llovet JM, Real MI, Montana X, Planas R, Coll S, Aponte J, et al: Arterial embolisation or chemoembolisation versus symptomatic treatment in patients with unresectable hepatocellular carcinoma: a randomised controlled trial. Lancet 359: 1734-1739, 2002.

7. Teicher BA: Hypoxia and drug resistance. Cancer Metast Rev 13: 39-68, 1994.

8. Brizel DM, Sibley GS, Prosnitz LR, Scher RL and Dewhirst MW: Tumor hypoxia adversely affects the prognosis of carcinoma of the head and neck. Int J Radiat Oncol Biol Phys 38: 285-289, 1997.

9. Overgaard J: Hypoxic radiosensitization: adored and ignored. J Clin Oncol 25: 4066-4074, 2007.

10. Wardman P: Chemical radiosensitizers for use in radiotherapy. Clin Oncol (R Coll Radiol) 19: 397-417, 2007.

11. Coleman CN, Wasserman TH, Urtasun RC, Halsey J, Hirst VK, Hancock S, et al: Phase I trial of the hypoxic cell radiosensitizer SR-2508: the results of the five to six week drug schedule. Int J Radiat Oncol Biol Phys 12: 1105-1108, 1986.

12. Tishler RB, Schiff PB, Geard CR and Hall EJ: Taxol: a novel radiation sensitizer. Int J Radiat Oncol Biol Phys 22: 613-617, 1992.

13. Watts ME, Dennis MF and Woodcock M: Uptake and additivity of the radiosensitizing effects of Ro 03-8799 and SR-2508 in mammalian cells in vitro. Br J Radiol 60: 1233-1235, 1987.

14. Jin C, Bai L and Guo G: Radiosensitization by the combination of SR-2508 and paclitaxel in hypoxic human tumor cells in vitro. J Radiat Res 48: 179-185, 2007.

15. Jin C, Bai L, Wu H, Tian F and Guo G: Radiosensitization of paclitaxel, etanidazole and paclitaxel+etanidazole nanoparticles on hypoxic human tumor cells in vitro. Biomaterials 28 : 3724-3730, 2007.

16. Seong J: Challenge and hope in radiotherapy of hepatocellular carcinoma. Yonsei Med J 50: 601-612, 2009.

17. Ma S, Jiao B, Liu X, Yi H, Kong D, Gao L, et al: Approach to radiation therapy in hepatocellular carcinoma. Cancer Treat Rev 36: 157-163, 2010.

18. Kerr SH and Kerr DJ: Novel treatments for hepatocellular cancer. Cancer Lett 286: 114-120, 2009.

19. Hudson CC, Liu M, Chiang GG, Otterness DM, Loomis DC, Kaper F, et al: Regulation of hypoxia-inducible factor 1alpha expression and function by the mammalian target of rapamycin. Mol Cell Biol 22: 7004-7014, 2002.

20. Bárdos JI and Ashcroft M: Negative and positive regulation of HIF-1: a complex network. Biochim Biophys Acta 1755: 107-120, 2005. 